\title{
On Cyclicity and Regularity of Commuting Matrices
}

\author{
Boris Shekhtman
}

\author{
Department of Mathematics and Statistics, University of South Florida, Tampa, FL 33620, United States \\ ${ }^{*}$ Corresponding Author: shekhtma@usf.edu
}

Copyright (C)2013 Horizon Research Publishing All rights reserved.

\begin{abstract}
It is well-known that the following properties of a matrix are equivalent: a matrix is non-derogatory if and only if is cyclic if and only if it is simple and if and only if it is 1-regular. In this article we attempt to extend these properties to a sequence of commuting matrices and examine the relation between them.
\end{abstract}

Keywords Commuting matrices, cyclicity, regularity, simplicity, non-derogatory sequences

\section{Introduction}

Sequences of commuting matrices play an important role in linear algebra (e.g. [9]) as well as its applications to numerical analysis (cf. $[1,19,18]$ ), algebra (cf. [5]), algebraic geometry (cf. $[3,12,13])$ and approximation theory (cf. $[4,14,16])$. The study of the irreducibility of the variety of commuting couples and triples of matrices was initiated by Motzkin and Taussky [11] and continued in [6], [7] and [8], among others. In this article we will extend some well-known properties of matrices to sequences of commuting matrices and examine their relations to each other.

Our starting point is the following standard fact from linear algebra (cf. [9])

For an $n \times n$ matrix $L$ with complex entries, the following four conditions are equivalent:

R) $L$ is regular, i.e., every eigenspace of $L$ is at most one-dimensional.

C) $L$ is cyclic, i.e., there exists a (cyclic) vector $v \in \mathbb{C}^{n}$ such that

$$
\operatorname{span}\left\{v, L v, \ldots, L^{n-1} v\right\}=\mathbb{C}^{n} .
$$

S) $L$ is simple, i.e., if $T$ commutes with $L$ then $T=$ $p(L)$ for some polynomial $p$.

D) $L$ is non-derogatory, i.e., the characteristic polynomial of $L$ is its minimal polynomial.

To what extend these equivalences extend to a sequences of commuting matrices? In this article we will examine the relationship between the four equivalent conditions for $d$-tuple of commuting matrices.

Here are some preliminaries: In what follows, $\mathcal{L}\left(\mathbb{C}^{n}\right)$ will stand for the algebra of linear operators on $\mathbb{C}^{n}$ or, equivalently, for the algebra of complex $n \times n$ matrices. $\mathbb{C}[\mathbf{x}]:=\mathbb{C}\left[x_{1}, \ldots, x_{d}\right]$ will denote the algebra of polynomials of $d$ variables with complex coefficients. For a subset $F \subset \mathbb{C}^{n}$ we let $[F]$ stand for the linear span of $F$.

Let $\mathbf{L}:=\left(L_{1}, \ldots, L_{d}\right)$ be a sequence of pairwise commuting $n \times n$ matrices with complex entries. A $d$-tuple $\lambda:=\left(\lambda_{1}, \ldots, \lambda_{d}\right) \in \mathbb{C}^{n}$ is called an eigentuple for $\mathbf{L}$ if there exists a non-zero vector $v \in \mathbb{C}^{n}$ such that $L_{j} v=\lambda_{j} v$ for all $j=1, \ldots, d$. Any such vector $v$ is called an eigenvector for $\mathbf{L}$ corresponding to an eigentuple $\lambda$. The set of all eigentuples for $\mathbf{L}$ is called the (joint) spectrum of $\mathbf{L}$ and denoted by $\sigma(\mathbf{L})$. It is wellknown and easy to see that $\sigma(\mathbf{L}) \neq \varnothing$ for any such $\mathbf{L}$. For $\lambda \in \sigma(\mathbf{L})$ the linear space

$$
V_{\lambda}:=\left\{v \in \mathbb{C}^{n}: L_{j} v=\lambda_{j} v, j=1, \ldots, d\right\} \subset \mathbb{C}^{n}
$$

is called an eigenspace for $\mathbf{L}$. A subspace $V \subset \mathbb{C}^{n}$ is $\mathbf{L}$-invariant if $L_{j} V \subset V$ for all $j=1, \ldots, d$. If $\mathbf{L}:=$ $\left(L_{1}, \ldots, L_{d}\right)$ we use $\mathbf{L}^{*}$ to denote the sequence of adjoint matrices $\left(L_{1}^{*}, \ldots, L_{d}^{*}\right)$. For $\lambda=\left(\lambda_{1}, \ldots, \lambda_{d}\right) \in \sigma(\mathbf{L})$ we use $\mathbf{L}_{\lambda}:=\left(L_{j}-\lambda_{j} I, j=1, \ldots, d\right)$. Finally we use $J_{\mathbf{L}}$ to denote the ideal of polynomials in $\mathbb{C}[\mathbf{x}]$ that annihilate L:

$$
J_{\mathbf{L}}:=\{p \in \mathbb{C}[\mathbf{x}]: p(\mathbf{L})=0\} .
$$

The following useful proposition is a part of the folklore:

Proposition 1.1. Let $U$ be an $\mathbf{L}^{*}$-invariant subspace of $\mathbb{C}^{n}$. Then $U^{\perp}$ is an $\mathbf{L}$-invariant subspace of $\mathbb{C}^{n}$.

Proof. For $v \in U^{\perp}$ and any $u \in U$ we have

$$
\left\langle L_{j} v, u\right\rangle=\left\langle v, L_{j}^{*} u\right\rangle=0
$$

since $L_{j}^{*} u \in U$ by our assumption. Hence $L_{j} v \in U^{\perp}$.

Definition 1.2. A $d$-tuple $\mathbf{L}:=\left(L_{1}, \ldots, L_{d}\right)$ of pairwise commuting $n \times n$ is called cyclic if there exists a (cyclic) vector $v \in \mathbb{C}^{n}$ such that $\{p(\mathbf{L}) v: p \in \mathbb{C}[\mathbf{x}]\}=\mathbb{C}^{n}$. 


\section{Cyclicity vs. regularity}

We start with a simple example (already used in [4], [10]) that shows that the equivalence of cyclicity and regularity fails for pairs of commuting matrices in both directions:

Example 2.1. First consider $\mathbf{L}=\left(L_{1}, L_{2}\right)$ on $\mathbb{C}^{3}$ given by

$$
L_{1}=\left[\begin{array}{lll}
0 & 0 & 0 \\
1 & 0 & 0 \\
0 & 0 & 0
\end{array}\right], L_{2}=\left[\begin{array}{lll}
0 & 0 & 0 \\
0 & 0 & 0 \\
1 & 0 & 0
\end{array}\right]
$$

This is a cyclic commuting pair with the cyclic vector $(1,0,0)$, yet $\sigma(\mathbf{L})=\{(0,0)\}$ and vectors $(0,1,0)$ and $(0,0,1)$ are common eigenvectors for $\mathbf{L}$. On the other hand $\mathbf{L}^{t}=\left(L_{1}^{t}, L_{2}^{t}\right)=\left(L_{1}^{*}, L_{2}^{*}\right)=\mathbf{L}^{*}$ given by

$$
L_{1}^{*}=\left[\begin{array}{ccc}
0 & 1 & 0 \\
0 & 0 & 0 \\
0 & 0 & 0
\end{array}\right], L_{2}^{*}=\left[\begin{array}{ccc}
0 & 0 & 1 \\
0 & 0 & 0 \\
0 & 0 & 0
\end{array}\right]
$$

is not cyclic (the range of each matrix is the same onedimensional subspace spanned by $(1,0,0))$, yet the only common eigenspace is one-dimensional, spanned by the vector $v=(1,0,0)$.

It took me a while to learn the lesson of this example: The cyclicity of a $d$-tuple of commuting matrices is related to the dimensions of the eigenspaces of the adjoint $d$-tuple rather then the matrices themselves. (Of course for one matrix it is a mute point.)

Theorem 2.2. A d-tuple $\mathbf{L}:=\left(L_{1}, \ldots, L_{d}\right)$ of commuting $N \times N$ matrices is cyclic iff the dimension of each eigenspace of $\mathbf{L}^{*}:=\left(L_{1}^{*}, \ldots, L_{d}^{*}\right)$ is at most one. In this case the sum of eigenvectors corresponding to distinct eigenvalues of $\mathbf{L}^{*}$ is a cyclic vector for $\mathbf{L}$.

Theorem 2.2 is an immediate corollary of more general Theorem 2.4 which requires a definition:

Definition 2.3. For a $d$-tuple $\mathbf{L}:=\left(L_{1}, \ldots, L_{d}\right)$ of commuting matrices a cyclicity cyc $(\mathbf{L})$ is the least integer $n$ such that there exist $n$ vectors $w_{1}, \ldots, w_{n}$ with

$$
+_{n=1}^{s}\left\{p(\mathbf{L}) w_{n}: p \in \mathbb{C}[\mathbf{x}]\right\}=\mathbb{C}^{n} .
$$

If cyc $(\mathbf{L})=n$, we will say that $\mathbf{L}$ is $n$-cyclic. Thus cyclic $d$-tuples are 1-cyclic.

Theorem 2.4. Let $\mathbf{L}:=\left(L_{1}, \ldots, L_{d}\right)$ be a sequence of commuting $n \times n$ matrices. Then the cyclicity $\operatorname{cyc}(\mathbf{L})$ is equal to the maximal dimension of eigenspaces of $\mathbf{L}^{*}$.

Proof. Let $\left\{v_{1}, \ldots v_{s}\right\}$ be the cyclic set for $s$-cyclic sequence $\mathbf{L}$ and let $u_{1}, \ldots, u_{s}, u_{s+1}$ be linearly independent eigenvectors that belong to the same eigentuple of $\mathbf{L}^{*}$. Then there exists a linear combination $u=\sum_{j=1}^{s+1} \alpha_{j} u_{j}$ orthogonal to $v_{1}, \ldots, v_{s}$ (more equations then the unknowns) and $[h]^{\perp}$ is a proper $\mathbf{L}$-invariant subspace containing $v_{1}, \ldots, v_{s}$. Contradiction.

Conversely, suppose that $U_{1}, \ldots, U_{m}$ are the eigenspaces of $\mathbf{L}^{*}$ that correspond to distinct eigentuples $\lambda_{1}, \ldots, \lambda_{m}$. Let $s:=\max \left\{\operatorname{dim} U_{j}, j=1, \ldots, m\right\}$. We will exhibit a set of vectors $\left\{w_{1}, \ldots, w_{s}\right\}$ which is a cyclic set for $\mathbf{L}$. For each $j=1, \ldots, m$ let $\left(u_{1, j}, \ldots, u_{s, j}\right)$ be vectors in $U_{j}$ such that $\left(u_{1, j}, \ldots, u_{k, j}\right)$ are linearly independent if $k \leq \operatorname{dim} H_{j}$ and $u_{k, j}=0$ if $k>\operatorname{dim} U_{j}$. Now for $n=1, \ldots, s$ we form vectors

$$
w_{n}:=\sum_{j=1}^{s} u_{n, j} .
$$

We claim that these vectors form a cyclic set for $\mathbf{L}$. Otherwise the space

$$
W:=+_{n=1}^{s}\left\{p(\mathbf{L}) w_{n}: p \in \mathbb{C}[\mathbf{x}]\right\}
$$

is a proper L-invariant subspace of $\mathbb{C}^{n}$ hence $W^{\perp}$ contains an eigenvector corresponding to some eigentuple, say $\lambda_{1}$, for $\mathbf{L}$. Let $p \in \mathbb{C}[\mathbf{x}]$ be such that $p\left(\lambda_{j}\right)=\delta_{1, j}$, for all $j=1, \ldots, m$. We have $p\left(\mathbf{L}^{*}\right) w_{n}=u_{n, 1}$; thus $W$ contains $U_{1}$ and cannot contain an eigenvector from $U_{1}$ orthogonal to it.

Remark 2.5. The second statement in Theorem 2.2 follows directly from the construction of the vectors $w_{n}$.

Next, I wish to examine the role that the quadratic polynomials in $\mathbf{L}^{*}$ play in the cyclicity structure of $\mathbf{L}$.

Since a nilpotent operator is not invertible, its rank is less then the dimension of the space. If $L_{1}$ and $0 \neq L_{2}$ are two commuting nilpotent operators the range of $L_{2}$ is $L_{1}$-invariant and $\left.L_{1}\right|_{\operatorname{ran} L_{2}}$ is still nilpotent; hence the $\operatorname{rank} L_{1} L_{2}<\operatorname{rank} L_{2}$ and

$$
\operatorname{dim} \operatorname{ker} L_{1} L_{2}>\operatorname{dim} \operatorname{ker} L_{2} .
$$

In particular for any nilpotent matrix $L \neq 0$,

$$
\operatorname{dim} \operatorname{ker} L^{2}>\operatorname{dim} \operatorname{ker} L .
$$

Similar result holds for the kernel of sequences of commuting nilpotent matrices; we just need to define the powers of $\mathbf{L}$ :

Definition 2.6. Let $\mathbf{L}:=\left(L_{1}, \ldots, L_{d}\right)$ be a sequence of commuting matrices. We define

$$
H_{m}(\mathbf{L})=\{p(\mathbf{L}): p \text { monomials of degree } m\} .
$$

Thus, for instance,

$$
H_{2}\left(L_{1}, L_{2}\right)=\left\{L_{1}^{2}, L_{1} L_{2}, L_{2}^{2}\right\} .
$$

Also notice that (ordering monomials of degree $m$ ) $H_{m}(\mathbf{L})$ is a sequence of commuting matrices.

Lemma 2.7. Let $0 \neq \mathbf{L}$ be a d-tuple of commuting nilpotent matrices. Then

$$
\operatorname{dim} \operatorname{ker} H_{2}(\mathbf{L})>\operatorname{dim} \operatorname{ker} \mathbf{L} .
$$

Proof. Since ker $\mathbf{L} \subset \operatorname{ker} L_{1}$ and by (2.3) above,

$$
\operatorname{dim} \operatorname{ker} L_{1}^{2}>\operatorname{dim} \operatorname{ker} L_{1} \geq \operatorname{dim} \operatorname{ker} \mathbf{L} .
$$

Assume that $k$ is the maximum number of quadratic monomials $p_{1}, \ldots, p_{k}$ such that

$$
\operatorname{dim} \operatorname{ker}\left(p_{j}(L), j=1, \ldots, k\right)>\operatorname{dim} \operatorname{ker} \mathbf{L} .
$$


Let

$$
V:=\operatorname{ker}\left(p_{j}(L), j=1, \ldots, k\right) .
$$

Suppose that a monomial $L_{i} L_{m}$ is missing from that list. Then $V$ is invariant for $L_{m}$ as well as $L_{i} L_{m}$ and $\operatorname{dim} \operatorname{ker}\left(\left.L_{i} L_{m}\right|_{V}\right)>\operatorname{dim} \operatorname{ker}\left(\left.L_{m}\right|_{V}\right) \geq \operatorname{dim} \operatorname{ker} \mathbf{L}$ since $\operatorname{ker} \mathbf{L} \subset V$. Hence

$\operatorname{dim} \operatorname{ker}\left(L_{i} L_{m}, p_{j}(L), j=1, \ldots, k\right)>\operatorname{dim} \operatorname{ker} \mathbf{L}$.

and thus $p_{1}, \ldots, p_{k}$ are all monomials of degree 2 .

The last lemma has an obvious generalization:

Proposition 2.8. Let $\mathbf{L}$ be a d-tuple of commuting nilpotent matrices. If for some $m \geq 1$ the set $H_{m}(\mathbf{L}) \neq$ $\{0\}$ then

$$
\operatorname{dim} \operatorname{ker} H_{m+1}(\mathbf{L})>\operatorname{dim} \operatorname{ker} H_{m}(\mathbf{L}) .
$$

Lemma 2.7 has an interesting corollary:

For a matrix $L$ define

$$
\sqrt{L}:=\left\{A: A^{2}=L\right\} .
$$

Corollary 2.9. For commuting matrices (2.2) from Example 2.1 , the sets $\sqrt{L_{1}^{*}}, \sqrt{L_{2}^{*}}$ are not empty yet for any $A_{i} \in \sqrt{L_{i}^{*}} A_{1}$ and $A_{2}$ do not commute.

Proof. It is easy to verify that

$$
\left(\begin{array}{lll}
0 & 0 & 1 \\
0 & 0 & 0 \\
0 & 1 & 0
\end{array}\right) \in \sqrt{L_{1}^{t}},\left(\begin{array}{lll}
0 & 1 & 0 \\
0 & 0 & 1 \\
0 & 0 & 0
\end{array}\right) \in \sqrt{L_{2}^{t}}
$$

hence $\sqrt{L_{1}}, \sqrt{L_{2}}$ are not empty.

If there exist commuting $A_{i} \in \sqrt{L_{i}^{*}}$ then $\operatorname{ker}\left(A_{1}^{2}, A_{2}^{2}\right)=\operatorname{ker}\left(L_{1}^{*}, L_{2}^{*}\right)$ would be at least two dimensional, which is false.

Here are another couple of corollaries of the lemma:

Corollary 2.10. If $0 \neq \mathbf{L}:=\left(L_{1}, \ldots, L_{d}\right)$ is a d-tuple of commuting matrices then for every $\lambda:=\left(\lambda_{1}, \ldots, \lambda_{d}\right) \in$ $\sigma\left(\mathbf{L}^{t}\right)$

$\operatorname{dim} \operatorname{ker}\left(\left(L_{i}-\lambda_{i} I\right)\left(L_{j}-\lambda_{j} I\right), i, j=1, \ldots, d\right) \geq \operatorname{dim} V_{\lambda}$.

Proof. The proof follows from Lemma 2.7 and blockdiagonalization of commuting matrices [10].

\section{Corollary 2.11.}

(i) $\mathbf{L}$ is simultaneously diagonalizable iff

$$
\begin{gathered}
\operatorname{dim} \operatorname{ker}\left(\mathbf{L}_{\lambda}, i=1, \ldots, d\right) \\
=\operatorname{dim} \operatorname{ker}\left(H_{2}\left(\mathbf{L}_{\lambda}\right), i=1, \ldots, d\right) .
\end{gathered}
$$

(ii) A d-tuple of commuting matrices has $N$ distinct eigentuples iff

$$
\operatorname{dim} \operatorname{ker}\left(\left(L_{i}-\lambda_{i} I\right)^{2}, i=1, \ldots, d\right)=1
$$

for every $\lambda:=\left(\lambda_{1}, \ldots, \lambda_{d}\right) \in \sigma\left(\mathbf{L}^{t}\right)$.

Next we want to address the situation when $\operatorname{dim} \operatorname{ker} H_{2}\left(\mathbf{L}^{*}\right)=2$. It follows from the Jordan form that one matrix $L$ is cyclic if and only if

$$
\operatorname{dim} \operatorname{ker} L^{2}=\operatorname{dim} \operatorname{ker}\left(L^{*}\right)^{2}=2 .
$$

Theorem 2.12. Let $N \geq 2$ and $0 \neq \mathbf{L}$. Then $\mathcal{A}(\mathbf{L})$ contains a cyclic matrix iff

$$
\operatorname{dim} \operatorname{ker} H_{2}\left(\mathbf{L}_{\lambda}^{*}\right)=2
$$

for every $\lambda: \in \sigma\left(\mathbf{L}^{*}\right)$.

Proof. It suffices to examine the nilpotent case. If $L \in$ $\mathcal{A}(\mathbf{L})$ is nilpotent and cyclic then, as follows from the Jordan form of $L, \operatorname{dim} \operatorname{ker} L^{2}=\operatorname{dim} \operatorname{ker}\left(L^{*}\right)^{2}=2$. This combined with Lemma 2.7 gives dim ker $H_{2}\left(\mathbf{L}^{*}\right)=2$.

For the converse, let $\operatorname{dim} \operatorname{ker} H_{2}\left(\mathbf{L}^{*}\right)=2$ then $\operatorname{dim} \operatorname{ker} H_{1}\left(\mathbf{L}^{*}\right)=1$ and $\mathbf{L}$ is cyclic. I claim that for $d$ commuting nilpotent matrices

$$
\mathbf{A}=\left(A_{1}, \ldots, A_{d}\right)
$$

with ker $\mathbf{A}=\left[v_{0}\right]$ and $\operatorname{dim} \operatorname{ker} H_{2}(\mathbf{A})=2$ at least one of $A_{j}$ is 1-regular. The proof is by induction on $d$. For $d=1$ the result follows from (2.3). Assume that it is true for $d-1$ and that (2.5) has no cyclic matrices. Then, by inductive assumption, there exist $u \in \operatorname{ker}\left(A_{2}, \ldots, A_{d}\right)$, $u \notin\left[v_{0}\right]$ and $w \in \operatorname{ker}\left(A_{1}, \ldots, A_{d-1}\right), w \notin\left[v_{0}\right]$. Let $k$ and $m$ be the least integers such that $A_{1}^{k} u=0$ and $A_{d}^{m} w=0$. I claim that
a) $0 \neq A_{1}^{k-1} u=A_{d}^{m-1} w \in\left[v_{0}\right]$
b) Vectors $v_{0}, A_{1}^{k-2} u, A_{d}^{m-2} w$ are linearly independent.
c) $v_{0}, A_{1}^{k-2} u, A_{d}^{m-2} w \in \operatorname{ker} H_{2}(\mathbf{A})$

The last two statements contradict $\operatorname{dim} \operatorname{ker} H_{2}(\mathbf{A})=$ 2 .

To prove a) we have

$$
A_{1}^{k-1} u \in \operatorname{ker} A_{1} \cap \operatorname{ker}\left(A_{2}, \ldots, A_{d}\right)=\operatorname{ker} \mathbf{A} .
$$

To prove b) assume that for some constants $\alpha, \beta$ and $\gamma$

$$
\alpha v_{0}+\beta A_{1}^{k-2} u+\gamma A_{d}^{m-2} w=0 .
$$

Then

$$
0=\alpha A_{1} v_{0}+\beta A_{1}^{k-1} u+\gamma A_{1} A_{d}^{m-2} w=\beta v_{0}
$$

hence $\beta=0$. The argument for $\gamma$ is the same. Finally c) follows from definitions of $u, w, k$ and $m$.

\section{Cyclic vs. simple and non- derogatory}

We start with a definition of a non-derogatory sequence of commuting matrices. Since the characteristic polynomial of an $n \times n$ matrix $L$ is of degree $n$ hence an equivalent definition of a non-derogatory matrix (cf. D) in the introduction) is $\operatorname{dim} \mathbb{C}[x] / J_{L}=n$. Thus the following seem to make sense:

Definition 3.1. A $d$-tuple $\mathbf{L}:=\left(L_{1}, \ldots, L_{d}\right)$ of commuting $n \times n$ matrices is non-derogatory if $\operatorname{dim}\left(\mathbb{C}[\mathbf{x}] / J_{\mathbf{L}}\right)=n$.

The definition of simplicity is straight forward:

Definition 3.2. A $d$-tuple $\mathbf{L}:=\left(L_{1}, \ldots, L_{d}\right)$ of commuting $n \times n$ matrices is simple if every $T$ that commutes with each $L_{j}$ is a polynomial in $\mathbf{L}$. 
Proposition 3.3. Let $\mathbf{L}:=\left(L_{1}, \ldots, L_{d}\right)$ be a d-tuple of commuting $N \times N$ matrices. Then, if $\mathbf{L}$ is cyclic it is simple and non-derogatory. Converse is not true.

Proof. Let $T$ commutes with every $L_{j}$ and let $v$ be a cyclic vector for $\mathbf{L}$. Then there exists a polynomial $q \in$ $\mathbb{C}[\mathbf{x}]$ such that $q(\mathbf{L}) v=T v$. Also for every vector $u \in \mathbb{C}^{n}$ there exists a polynomial $p_{u} \in \mathbb{C}[\mathbf{x}]$ such that $p_{u}(\mathbf{L}) v=$ $u$. We have

$$
\begin{gathered}
T u=T p_{u}(\mathbf{L}) v=p_{u}(\mathbf{L}) T v=p_{u}(\mathbf{L}) q(\mathbf{L}) v \\
=q(\mathbf{L}) p_{u}(\mathbf{L}) v=q(\mathbf{L}) u
\end{gathered}
$$

hence $T=q(\mathbf{L})$.

To prove that a cyclic sequence is non-derogatory we let, once again, $v$ be a cyclic vector for $\mathbf{L}$ and define a mapping

$$
\begin{aligned}
\varphi: \mathbb{C}[\mathbf{x}] & \rightarrow \mathbb{C}^{n} \\
f & \rightarrow f(\mathbf{L}) v
\end{aligned}
$$

Since $\mathbf{L}$ is cyclic, $\varphi$ is onto and by the fundamental theorem of homomorphisms $\mathbb{k}[\mathbf{x}] / \operatorname{ker} \varphi$ is isomorphic to $\mathbb{C}^{n}$ hence $\operatorname{dim} \mathbb{k}[\mathbf{x}] / \operatorname{ker} \varphi=n$. It remains to show that $\operatorname{ker} \varphi=J_{\mathbf{L}}$. Clearly if $f(\mathbf{L})=0$ then $f(\mathbf{L}) v=0$. Now assume that $f \in \operatorname{ker} \varphi$, i.e., $f(\mathbf{L}) v=0$. Since for every $u \in \mathbb{C}$ there exists a polynomial $p_{u} \in \mathbb{C}[\mathbf{x}]$ such that $p_{u}(\mathbf{L}) v=u$ we have

$$
f(\mathbf{L}) u=f(\mathbf{L}) p_{u}(\mathbf{L}) v=p_{u}(\mathbf{L}) f(\mathbf{L}) v=0
$$

and $f \in J_{\mathbf{L}}$.

To show that the converse fails, we, once more, consider the matrices $\mathbf{L}^{*}$ from Example 2.1. Since $\mathbf{L}$ is cyclic $\operatorname{dim}\left(\mathbb{C}\left[x_{1}, x_{2}\right] / J_{\mathbf{L}}\right)=3$ but $J_{\mathbf{L}}=J_{\mathbf{L}^{*}}$ hence $\mathbf{L}^{*}$ is nonderogatory yet not cyclic. Similarly, if $T$ commutes with $L_{1}^{*}$ and $L_{2}^{*}$ then $T^{*}$ commutes with $L_{1}$ and $L_{2}$ and, since $\left(L_{1}, L_{2}\right)$ is cyclic, it follows that there exists a polynomial $q$ such that $T^{*}=q\left(L_{1}, L_{2}\right)$. Hence $T=\bar{q}\left(L_{1}^{*}, L_{2}^{*}\right)$ and $\left(L_{1}^{*}, L_{2}^{*}\right)$ is simple yet non-derogatory.

\section{Simple vs. non-derogatory}

In this section we will that neither one of the two conditions implies the other.

First we will show that there exists a simple sequence of commuting matrices which is derogatory. In fact we will construct a simple sequence $\mathbf{L}$ such that $\operatorname{dim}\left(\mathbb{C}[\mathbf{x}] / J_{\mathbf{L}}\right)<n$ and a simple sequence $\mathbf{L}$ such that $\operatorname{dim}\left(\mathbb{C}[\mathbf{x}] / J_{\mathbf{L}}\right)>n$.

For the first construction, recall Courter's example ([2], cf. also [17]) of a commutative subalgebra $\mathcal{A} \subset$ $L\left(\mathbb{C}^{14}\right)$ of dimension 13 which is maximal, i.e., such every matrix that commutes matrices in is in $\mathcal{A}$. Let $\mathbf{L}:=\left(L_{1}, \ldots, L_{13}\right)$ be a basis in $\mathcal{A}$. Then, by maximality, every matrix that commutes with matrices in $\mathbf{L}$ is a (linear homogeneous) polynomial of $\mathbf{L}$. Hence $\mathbf{L}$ is simple. On the other hand

$$
\operatorname{dim}\left(\mathbb{C}\left[x_{1}, \ldots, x_{13}\right] / J_{\mathbf{L}}\right)=\operatorname{dim} \mathcal{A}=13<14 .
$$

To see this, consider the 13 -dimensional space $H \subset$ $\mathbb{C}\left[x_{1}, \ldots, x_{13}\right]$ of linear homogeneous polynomial. On the other hand for every polynomial $p \in \mathbb{C}\left[x_{1}, \ldots, x_{13}\right]$ the matrix $p(\mathbf{L})$ commutes with $\mathbf{L}$, hence there exists $h \in H$ such that $p(\mathbf{L})=h(\mathbf{L})$, hence $p=h+(p-h)$ where $h \in H$ and $(p-h) \in J_{\mathbf{L}}$, i.e.,

$$
H+J_{\mathbf{L}}=\mathbb{C}\left[x_{1}, \ldots, x_{13}\right] .
$$

Since $\left(L_{1}, \ldots, L_{13}\right)$ are linearly independent, it follows that $H \cap J_{\mathbf{L}}=\{0\}$, and the sum in (4.2) is a direct sum which proves (4.1).

For the second construction consider a sequence $\mathbf{L}$ of four matrices

$$
\mathbf{L}=\left(\left[\begin{array}{llll}
0 & 0 & 1 & 0 \\
0 & 0 & 0 & 0 \\
0 & 0 & 0 & 0 \\
0 & 0 & 0 & 0
\end{array}\right],\left[\begin{array}{llll}
0 & 0 & 0 & 1 \\
0 & 0 & 0 & 0 \\
0 & 0 & 0 & 0 \\
0 & 0 & 0 & 0
\end{array}\right],\left[\begin{array}{llll}
0 & 0 & 0 & 0 \\
0 & 0 & 1 & 0 \\
0 & 0 & 0 & 0 \\
0 & 0 & 0 & 0
\end{array}\right],\left[\begin{array}{llll}
0 & 0 & 0 & 0 \\
0 & 0 & 0 & 1 \\
0 & 0 & 0 & 0 \\
0 & 0 & 0 & 0
\end{array}\right]\right) \text {. }
$$

It can be verified by direct computations that every matrix that commutes with $\mathbf{L}$ is a (linear) polynomial in $\mathbf{L}$, hence $\mathbf{L}$ is simple. On the other hand the identity matrix $I$ is not a linear combination of $L_{1}, \ldots, L_{4}$, yet in the algebra

$$
\mathcal{A}:=\left\{p(\mathbf{L}): p \in \mathbb{C}\left[x_{1}, \ldots, x_{4}\right)\right\} .
$$

Combined with the fact that $L_{j} L_{k}=0$ for all $j, k=$ $1, \ldots, 4$ we conclude that $\mathcal{A}$ is the space of linear polynomials in four variable, its dimension is $5>4$ and, by the same argument as above,

$$
\operatorname{dim}\left(\mathbb{C}\left[x_{1}, \ldots, x_{4}\right] / J_{\mathbf{L}}\right)=\operatorname{dim} \mathcal{A}=5<4 .
$$

For the next example consider the sequence $\tilde{\mathbf{L}}=$ $\left(L_{1}, L_{2}, L_{3}\right)$ consisting of the first three matrices in first (4.3). As was mentioned earlier the pairwise product of these matrices are zero, hence

$$
\begin{aligned}
\mathcal{A}:= & \left\{p(\tilde{\mathbf{L}}): p \in \mathbb{C}\left[x_{1}, x_{2}, x_{3}\right]\right\} \\
= & \operatorname{span}\left\{I, L_{1}, L_{2}, L_{3}\right\}
\end{aligned}
$$

which is 4-dimensional, hence $\operatorname{dim}\left(\mathbb{C}\left[x_{1}, x_{2}, x_{3}\right] / J_{\tilde{\mathbf{L}}}\right)=$ 4 and $\tilde{\mathbf{L}}$ is non-derogatory. The fourth matrix in (4.3) commutes with the other three but is not a linear combination of the four matrices in the right-hand side of (4.4). The equality (4.4) thus implies that $L_{4}$ is not a polynomial in $\left(I, L_{1}, L_{2}, L_{3}\right)$ and hence $\tilde{\mathbf{L}}$ is not simple.

\section{Similarity of commuting se- quences}

We finish this article with a remark about similarity of commuting $d$-tuples.

Definition 5.1. Two $d$-tuples $\mathbf{L}:=\left(L_{1}, \ldots, L_{d}\right)$ and $\mathbf{T}:=\left(T_{1}, \ldots, T_{d}\right)$ are similar $(\mathbf{L} \sim \mathbf{T})$ if there exists an invertible matrix $S$ such that

$$
T_{j}=S L_{j} S^{-1}
$$

for all $j=1, \ldots, d$.

It is easy to see from the Jordan for that a matrix is it is always similar to its transpose. Example 2.1 shows that it is not the case for a $d$-tuples: $\mathbf{L}:=\left(L_{1}, L_{2}\right)$ is not similar to its transpose $\mathbf{L}^{t}:=\left(L_{1}^{t}, L_{2}^{t}\right)$. The following observation was proven in [15]:

Proposition 5.2. A cyclic commuting d-tuple $\mathbf{L}$ is similar to a commuting d-tuple $\mathbf{T}$ iff $J_{\mathbf{L}}=J_{\mathbf{T}}$ and $\mathbf{T}$ is cyclic. We will now present a direct proof of this fact. 
Proof. Let $u$ be a cyclic vector for $\mathbf{L}$ and $v$ be a cyclic vector for T. Define a mapping $S: \mathbb{C}^{N} \rightarrow \mathbb{C}^{N}$ by letting

$$
S(p(\mathbf{L}) u)=p(\mathbf{T}) v
$$

for every $p \in \mathbb{C}[\mathbf{x}]$. Since $u$ is a cyclic vector for $\mathbf{L},\{p(\mathbf{L}) u, p \in \mathbb{C}[\mathbf{x}]\}=\mathbb{C}^{N}$ hence $S$ is defined for all $w \in \mathbb{C}^{N}$. To show that $S$ is well-defined assume that $p_{1}(\mathbf{L}) u=p_{2}(\mathbf{L}) u$. Then $\left(p_{1}(\mathbf{L})-p_{2}(\mathbf{L})\right) u=0$ and hence $p_{1}-p_{2} \in J_{\mathbf{L}}$. By assumption this implies $p_{1}-p_{2} \in J_{\mathbf{T}}$ thus $p_{1}(\mathbf{T})-p_{2}(\mathbf{T})=0$. In particular $p_{1}(\mathbf{T}) v=p_{2}(\mathbf{T}) v$ and $S$ is well defined and linear. Since $v$ is a cyclic vector for $\mathbf{T}$ the map $S$ is onto hence $S$ is invertible. Now let $w \in \mathbb{C}^{N}$. Then there exists a polynomial $p$ such that $w=p(\mathbf{L}) u$. Hence

$$
\begin{gathered}
S L_{j} w=S L_{j} p(\mathbf{L}) u=S\left(x_{j} p\right)(\mathbf{L}) \mathbf{u} \\
=\left(x_{j} p\right)(\mathbf{T}) \mathbf{v}=T_{j} p(\mathbf{T}) \mathbf{v}=T_{j} S p(\mathbf{L}) u=T_{j} S w .
\end{gathered}
$$

Hence $S L_{j}=T_{j} S$ for every $j$ and $\mathbf{L} \sim \mathbf{T}$.

In particular a cyclic commuting $d$-tuple $\mathbf{L}$ is similar to its transpose if and only if $\mathbf{L}^{t}$ is cyclic.

Problem 5.3. What is a good criteria for general $d$ tuple of commuting matrices to be similar to its transpose? Is it sufficient to assume that $\operatorname{cyc}(\mathbf{L})=\operatorname{cyc}\left(\mathbf{L}^{t}\right)$ ?

\section{Acknowledgement}

I would like to thank the referee for the kind words and many useful suggestions.

\section{REFERENCES}

[1] W. Auzinger and H. J. Stetter. An elimination algorithm for the computation of all zeros of a system of multivariate polynomial equations. In Numerical mathematics, Singapore 1988, volume 86 of Internat. Schriftenreihe Numer. Math., pages 11-30. Birkhäuser, Basel, 1988.

[2] R. C. Courter. The dimension of maximal commutative subalgebras of $K_{n}$. Duke Math. J., 32:225-232, 1965.

[3] David A. Cox. Solving equations via algebras. In Solving polynomial equations, volume 14 of Algorithms Comput. Math., pages 63-123. Springer, Berlin, 2005.

[4] C. de Boor and B. Shekhtman. On the pointwise limits of bivariate Lagrange projectors. Linear Algebra Appl., 429(1):311-325, 2008.
[5] Murray Gerstenhaber. On nilalgebras and linear varieties of nilpotent matrices. III. Ann. of Math. (2), 70: 167-205, 1959.

[6] Robert M. Guralnick. A note on commuting pairs of matrices. Linear and Multilinear Algebra, 31(1-4):71$75,1992$.

[7] Robert M. Guralnick and B. A. Sethuraman. Commuting pairs and triples of matrices and related varieties. Linear Algebra Appl., 310(1-3):139-148,2000.

[8] Yongho Han. Commuting triples of matrices. Electron. J. Linear Algebra, 13:274-343, 2005.

[9] Roger A. Horn and Charles R. Johnson. Matrix Analysis. Cambridge University Press, Cambridge, 1985.

[10] Tom McKinley and Boris Shekhtman. On simultaneous block-diagonalization of cyclic sequences of commuting matrices. Linear Multilinear Algebra, 58(1-2):245-256, 2010.

[11] T. S. Motzkin and Olga Taussky. Pairs of matrices with property L. II. Trans. Amer. Math. Soc., 80:387-401, 1955.

[12] Hiraku Nakajima. Lectures on Hilbert schemes of points on surfaces, volume 18 of University Lecture Series. American Mathematical Society, Providence, RI, 1999.

[13] Lorenzo Robbiano. Zero-dimensional ideals or the inestimable value of estimable terms. In Constructive algebra and systems theory, volume 53 of Verh. Afd. Natuurkd. 1. Reeks. K. Ned. Akad. Wet., pages 95-114. R.Neth. Acad. Arts Sci., Amsterdam, 2006.

[14] Boris Shekhtman. Ideal interpolation: translations to and from algebraic geometry. In Approximate commutative algebra, Texts Monogr. Symbol. Comput., pages 163-192. SpringerWienNewYork, Vienna, 2009.

[15] Boris Shekhtman. Some tidbits on ideal projectors, commuting matrices and their applications. Electron. Trans. Numer. Anal., 36:17-26, 2009/10.

[16] Boris Shekhtman. A taste of ideal projectors. J. Concr. Appl. Math., 8(1):125-149, 2010.

[17] Young Kwon Song. On the maximal, commutative, subalgebras of 14 by 14matrices. Comm. Algebra, 25(12): 3823-3840, 1997.

[18] Hans J. Stetter. Matrix eigenproblems at the heart of polynomial system solving. SIGSAM Bull., 30(4):22-25, 1995.

[19] Hans J. Stetter. Numerical polynomial algebra. Society for Industrial and Applied Mathematics (SIAM), Philadelphia,PA, 2004. 